\title{
WEIGHTED TRANSLATION SEMIGROUPS
}

\author{
MARY R. EMBRY AND ALAN LAMBERT ${ }^{(1)}$
}

1. Introduction. The semigroup of translations on $L^{2}\left(\mathbf{R}_{+}\right)$is in many ways a continuous analogue of the unilateral shift on $\ell_{+}{ }^{2}$. In this article we examine a class of semigroups which to a great extent carry this analogy to weighted unilateral shifts. In section 2 we establish the notation used throughout the paper and study some of the basic structure of the semigroups under investigation. In particular, we show precisely when two such semigroups are unitarily equivalent and when such a semigroup is strongly continuous.

In section 3 we are concerned with special classes of semigroups, e.g., hyponormal and subnormal semigroups. We characterize which of the semigroups under investigation are hyponormal in terms of their generators and in terms of the symbols used in defining the semigroups. We then offer an example of a hyponormal, nonsubnormal semigroup.

2. Preliminaries. Let $\mathbf{R}_{+}$be the set of nonnegative real numbers and let $L^{2}=L^{2}\left(\mathbf{R}_{+}\right)$be the Hilbert space of (equivalence classes of) square integrable (Lebesgue) measurable functions from $R_{+}$into $\mathrm{C}$. Set $B\left(L^{2}\right)$ equal to the algebra of all (bound, linear) operators from $L^{2}$ into $L^{2}$. A family $\left\{S_{t}: t \in \mathbf{R}_{+}\right\}$in $B\left(L^{2}\right)$ is a semigroup if $S_{0}=I$, the identity operator, and for all $t$ and $s$ in $\mathbf{R}_{+}, S_{t} S_{s}=S_{t+s} . \quad\left\{S_{t}\right\}$ is a strongly continuous semigroup if $\left\{S_{t}\right\}$ is a semigroup and for each $f$ in $L^{2}$ the mapping $t \rightarrow S_{t} f$ is continuous from $\mathbf{R}_{+}$into $L^{2}$. It is well known that $\left\{S_{t}\right\}$ is strongly continuous on $\mathbf{R}_{+}$if and only if $\left\{\mathrm{S}_{t}\right\}$ is strongly continuous at $t=0$. Perhaps the most widely studied semigroup in $B\left(L^{2}\right)$ is the translation semigroup $\left\{U_{t}\right\}$ defined by $\left(U_{t} f\right)(x)=f(x-t)$ for $x \geqq t$ and 0 otherwise. More generally, suppose $\phi$ is a measurable, almost everywhere non-zero function from $\mathbf{R}_{+}$into $\mathbf{C}$ such that for each fixed $t$ the function $\phi_{t}$ defined by

$$
\phi_{t}(x)=\left\{\begin{array}{cl}
\frac{\phi(x)}{\phi(x-t)} ; & x \geqq t \\
0 ; & x<t
\end{array}\right.
$$

Received by the editors on August 27, 1975, and in revised form on February 16, 1976.

(1)Research supported by a University of North Carolina at Charlotte summer research grant. 
is essentially bounded. Define $S_{t}$ on $L^{2}$ by $S_{t} f(x)=\phi_{t}(x)\left(U_{t} f\right)(x)$. One may verify without difficulty that $\left\{S_{t}\right\}$ is a semigroup in $B\left(L^{2}\right)$. For simplicity throughout this article, we shall assume that $\phi$ is continuous on $\mathbf{R}_{+}$. Also, in order to avoid notational millstones, we shall assume that all expressions involving a symbol such as $g(x-t)$ are 0 for $x<t$.

LEMMA 2.1. With $\phi$ and $S_{t}$ as above, $\left\{S_{t}\right\}$ is strongly continuous if and only if there exist numbers $M$ and $w$ in $\mathrm{R}$ so that for each $t$ in $\mathbf{R}_{+}$

$$
\underset{x \in \mathrm{R}_{+}}{\operatorname{ess} \sup }\left|\frac{\phi(x+t)}{\phi(x)}\right| \leqq M e^{w t} .
$$

Proof. It follows from [3; p. 619] that if $\left\{S_{t}\right\}$ is strongly continuous then there exist $M$ and $w$ so that (1) holds. To prove the converse suppose first that (1) holds with $w=0$, i.e., $\left\{\phi_{t}: t \in \mathbf{R}_{+}\right\}$is uniformly bounded in $L^{\infty}$. As a simple calculation verifies, $\left\|S_{t}\right\|=\left\|\phi_{t}\right\|_{\infty}$ so $\left\{S_{t}\right\}$ is uniformly bounded. Thus in order to prove that $\left\{S_{t}\right\}$ is strongly continuous it suffices to show that $\lim _{t \rightarrow 0} S_{t} f=f$ for all $f$ in a dense subset of $L^{2}$. For this dense subset we choose the set of continuous functions of compact support. Let $f$ be such a function. Then

$$
\left\|S_{t} f-f\right\|^{2}=\int_{0}^{\infty}\left|\frac{\phi(x)}{\phi(x-t)} f(x-t)-f(x)\right|^{2} d x .
$$

Now for any $x$ and $t|\phi(x) / \phi(x-t) f(x-t)-f(x)|^{2} \leqq(M+1)^{2}$ $\|f\|_{\infty}^{2}$ and for each fixed $x \lim _{t \rightarrow 0}|\phi(x)| \phi(x-t) f(x-t)-f(x) \mid$ $=0$. Thus by the bounded convergence theorem $\lim _{t \rightarrow 0}\left\|S_{t} f-f\right\|=0$.

In general, suppose (1) holds for some $M$ and $w$ in $\mathbf{R}$. Define $\rho$ on $\mathbf{R}_{+}$by $\rho(x)=e^{-w x} \phi(x)$. Then for all $t$ and $x \geqq t$,

$$
\begin{aligned}
\left|\frac{\rho(x)}{\rho(x-t)}\right| & =\left|\frac{e^{-w x} \phi(x)}{e^{-w(x-t)} \phi(x-t)}\right| \\
& =e^{-w t}\left|\frac{\phi(x)}{\phi(x-t)}\right| \leqq M .
\end{aligned}
$$

Moreover, if $T_{t}=e^{-w t} S_{t}$, then

$$
\begin{aligned}
\left(T_{t} f\right)(x) & =e^{-w t} \frac{\phi(x)}{\phi(x-t)} f(x-t) \\
& =\frac{\rho(x)}{\rho(x-t)} f(x-t) .
\end{aligned}
$$


Thus $\left\{T_{t}\right\}$ and hence $\left\{S_{t}=e^{w t} T_{t}\right\}$ are strongly continuous. This completes the proof of the lemma.

Often the translation semigroup $\left\{U_{t}\right\}$ is viewed as a continuous analogue of the discrete semigroup $\left\{U^{n}: n=0,1, \cdots\right\}$ where $U$ is the unilateral shift. We shall show that the semigroups $\left\{S_{t}\right\}$ defined above are in many ways a continuous analogue of unilateral weighted shifts. For general information about weighted shifts the reader is directed to [4] and [7]. We shall refer to the type of semigroups appearing in Lemma 2.1 as weighted translation semigroups with symbol $\phi$.

Let $\left\{S_{t}\right\}$ be a weighted translation semigroup (henceforth w.t.s.) with symbol $\phi$. For each $t \geqq 0$ and each nonnegative integer $n$ let $X_{n}{ }^{(t)}$ be the characteristic function of the interval $[n t,(n+1) t]$. Set $M_{t}$ equal to the closed linear span of $\left\{\phi \cdot X_{n}{ }^{(t)}: n \geqq 0\right\}$. Let $\lambda_{n}{ }^{(t)}=$ $\left[\int_{n t}^{(n+1) t}|\phi(x)|^{2} d x\right]^{1 / 2}$.

LEMMA 2.2. For each $t, M_{t}$ is invariant under $S_{t}$, and $A_{t}$, the restriction of $S_{t}$ to $M_{t}$, is a weighted shift with weight sequence $\left\{\lambda_{0}{ }^{(t)}\right.$, $\left.\lambda_{1}{ }^{(t)} / \lambda_{0}{ }^{(t)}, \lambda_{2}{ }^{(t)} / \lambda_{1}{ }^{(t)}, \cdots\right\}$.

Proof. Fix $t \geqq 0$. For each nonnegative integer $n$ and each $x$ in $\mathbf{R}_{+}$,

$$
\begin{aligned}
\left(S_{t} \phi X_{n}{ }^{(t)}\right)(x) & =\frac{\phi(x)}{\phi(x-t)} \phi(x-t) X_{n}^{(t)}(x-t) \\
& =\phi(x) X_{n+1}^{(t)}(x) .
\end{aligned}
$$

Thus $M_{t}$ is invariant for $S_{t}$. Moreover, note that $\left\{e_{n}{ }^{(t)}=\left(1 / \lambda_{n}{ }^{(t)}\right)\right.$ $\left.\phi X_{n}{ }^{(t)}\right\}$ is an orthonormal basis for $M_{t}$ and

$$
S_{t} e_{n}^{(t)}=\frac{\lambda_{n+1}^{(t)}}{\lambda_{n}^{(t)}} e_{n+1}^{(t)}
$$

This completes the proof.

If $\left\{S_{t}\right\}$ is a w.t.s. with symbol $\phi$ and $A$ is any operator on $L^{2}$ then for each $t \geqq 0$ and each nonnegative integer $j$ there is a sequence of measurable functions $\left\{f_{i j}^{(t)}\right\}$ so that

$$
\operatorname{A\phi }_{j}(t)=\sum_{i=0}^{\infty} f_{i j}^{(t)} X_{i}^{(t)}
$$

We offer next a result which both in statement and proof is related to $[7$, p. 5]. 
Lemma 2.3. Suppose $\left\{\mathrm{S}_{t}\right\}$ and $\left\{T_{t}\right\}$ are w.t.s. with symbols $\phi$ and $\rho$ respectively. Let $A$ be in $B\left(L^{2}\right)$ and let $\left\{f_{i j}^{\prime(t)}\right\}$ be defined by (2). If $\mathrm{AS}_{t}=T_{t} A$ for all $t$ then

$$
f_{i j}^{(t)}= \begin{cases}0 ; & i<j \\ \rho U_{j t}\left(1 / \rho f_{i-j 0}^{(t)}\right) ; & i \geqq j .\end{cases}
$$

Proof. Suppose $A S_{t}=T_{t} A$ for each $t$. Then

$$
\begin{aligned}
\operatorname{AS}_{t} \phi X_{j}{ }^{(t)} & =A \phi X_{j+1}^{(t)} \\
& =\sum_{i=0}^{\infty} f_{i}^{(t)}{ }_{j+1} X_{i}{ }^{(t)}
\end{aligned}
$$

and

$$
\begin{aligned}
\left(T_{t} A \phi X_{j}^{(t)}\right)(x) & =\sum_{i=0}^{\infty}\left(T_{t} f_{i j}^{(t)} X_{i}^{(t)}\right)(x) \\
& =\sum_{i=0}^{\infty} \frac{\rho(x)}{\rho(x-t)} f_{i j}^{(t)}(x-t) X_{i}^{(t)}(x-t) \\
& =\left(\sum_{i=0}^{\infty}\left[\rho L_{t} \frac{1}{\rho} f_{i j}^{(t)}\right] X_{i+1}^{(t)}\right)(x) .
\end{aligned}
$$

Thus $f_{0}\left({ }_{j+1}=0\right.$ and

$$
f_{i+1 j+1}^{(t)}=\rho\left(L_{t} \frac{1}{\rho} f_{i j}^{(t)}\right) .
$$

A simple (and omitted) inductive argument completes the proof.

Corollary 2.4. If $\left\{S_{t}\right\},\left\{T_{t}\right\}$ and $A$ are as in Lemma 2.3 then for every $a \geqq 0 L^{2}([a, \infty))$ is invariant under $A$.

Proof. The linear span of $\left\{\phi X_{n}^{(t)}: n t \geqq a\right\}$ is dense in $L^{2}([a, \infty))$. Since $A \phi X_{n}{ }^{(t)}=\sum_{i=n}^{\infty}\left[\rho U_{n t}\left((1 / \rho) f_{i-n}^{(t)} 0\right)\right] \cdot X_{i}^{(t)}$ is a member of $L^{2}([a, \infty))$, the assertion is proved.

We are now able to classify weighted translation semigroups up to unitary equivalence.

THEOREM 2.5. Let $\left\{\mathrm{S}_{t}\right\}$ and $\left\{T_{t}\right\}$ be weighted translation semigroups with symbols $\phi$ and $\rho$ respectively. Then $\left\{\mathrm{S}_{t}\right\}$ is unitarily equivalent to $\left\{T_{t}\right\}$ if and only if the function $|\phi| \rho \mid$ is constant on $\mathbf{R}_{+}$. 
Proof. Suppose first that $|\phi| \rho \mid=c$ for some constant $c$. Define $W$ on $L^{2}$ by $(W f)(x)=c(\rho(x) / \phi(x)) f(x)$. It is clear that $W$ is a unitary operator. Moreover,

and

$$
\begin{aligned}
\left(W S_{t} f\right)(x) & =c \frac{\rho(x)}{\phi(x)} \frac{\phi(x)}{\phi(x-t)} \quad f(x-t) \\
& =c \frac{\rho(x)}{\phi(x-t)} \quad f(x-t)
\end{aligned}
$$

$$
\begin{aligned}
\left(T_{t} W f\right)(x) & =\frac{\rho(x)}{\rho(x-t)} \cdot c \frac{\rho(x-t)}{\phi(x-t)} f(x-t) \\
& =c \frac{\rho(x)}{\phi(x-t)} \quad f(x-t) .
\end{aligned}
$$

Thus for every $t, W S_{t}=T_{t} W$.

Conversely, suppose that there is a unitary operator $W$ so that $W S_{t}=T_{t} W$ for all $t \geqq 0$. By applying Corollary 2.4 and then reversing the roles of $S_{t}$ and $T_{t}$ and replacing $W$ by $W^{-1}$ in Corollary 2.4 one sees that for every finite interval $(a, b), L^{2}(a, b)$ is invariant for $W$. It follows immediately that $L^{2}(E)$ is invariant under $W$ for every open set $E$. Let $F$ be any measurable set and suppose $f$ is an $L^{2}$ function with support in $F$. There is a decreasing sequence $\left\{E_{n}\right\}$ of open sets such that the measure of $E_{n}-F$ is no greater than $1 / n$ and each $E_{n} \supset F$. But then $f$ is supported in $E_{n}$ and so

$$
\begin{aligned}
\|W f\|^{2} & =\int_{E_{n}}|(W f)(x)|^{2} d x \\
& =\int_{F}|(W f)(x)|^{2} d x+\int_{E_{n}-F}|(W f)(x)|^{2} d x .
\end{aligned}
$$

Hence $\quad \int_{\mathbf{R}+}|(W f)(x)|^{2} d x=\int_{F}|(W f)(x)|^{2} d x$ so that $W f=0$ a.e. off $F$. Now, the lattice of invariant subspaces for the maximal abelian von Neumann algebra of multiplications by $L^{\infty}$ functions is precisely $\left\{L^{2}(F): F\right.$ measurable $\}$. Since every von Neumann algebra is generated by its projections, we see that there is an $L^{\infty}$ function $w$ (of modulus one a.e.) so that $W f=w \cdot f$ for all $f$ in $L^{2}$. Now, for each $f$ in $L^{2}$, each $t \geqq 0$, and almost every $x \geqq t$,

$$
\begin{aligned}
w(x) \frac{\phi(x)}{\phi(x-t)} f(x-t) & =\left(W S_{t} f\right)(x) \\
& =\left(T_{t} W f\right)(x) \\
& =\frac{\rho(x)}{\rho(x-t)} w(x-t) f(x-t) .
\end{aligned}
$$


Thus $|\rho(x)| \rho(x-t)|=| \phi(x)|\phi(x-t)|$ for each $t \geqq 0$ and for almost every $x \geqq t$. But since $\rho$ and $\phi$ are continuous we have the last equality above holding for all $t$ and for all $x \geqq t$. Thus for every $x \geqq 0$ $|\rho(x) / \phi(x)|=|\rho(0) / \phi(0)|$, completing the proof.

REMARK 1. It follows immediately from Theorem 2.5 that the weighted translation semigroups with symbols $\phi$ and $|\phi|$ are unitarily equivalent. For this reason we shall assume throughout the remainder of this paper that all w.t.s. symbols are positive valued.

3. Hyponormal and Subnormal Weighted Translation Semigroups. In this section we restrict our attention to weighted translation semigroups with symbol $\phi$ which satisfy special operator theoretic conditions. A semigroup $\left\{S_{t}\right\}$ is paranormal, hyponormal, subnormal, quasinormal or normal if each $S_{t}$ has the given property. (An operator $A$ is paranormal if $\|A f\|^{2} \leqq\left\|A^{2} f\right\|\|f\|$ for all $f$, hyponormal if $\left\|A^{*} \mathrm{f}\right\|$ $\leqq\|A f\|$ for all $f$, subnormal if $A$ is the restriction of a normal operator to an invariant subspace, quasinormal if $A$ commutes with $A^{*} A$; and normal if $A$ commutes with $A^{*}$.) Each of these properties is preserved under unitary equivalence and thus by Theorem 2.5 we may assume $\phi$ to be positive.

Throughout the section we assume that $\left\{S_{t}\right\}$ is a semigroup with continuous symbol $\phi$. As in section 2 we let $M_{t}$ be the closed linear span of the functions $\phi X_{n}{ }^{(t)}$ where $X_{n}{ }^{(t)}$ is the characteristic function of the interval $[n t,(n+1) t]$. By Lemma 2.2 we know that $M_{t}$ is an invariant subspace for $S_{t}$ and that $S_{t} / M_{t}$ is a weighted shift. Consideration of these weighted shifts gives us criteria by which $\left\{\mathrm{S}_{t}\right\}$ is judged to be subnormal or hyponormal.

THeOREM 3.1. $\left\{S_{t}\right\}$ is subnormal if and only if $\left\{S_{t} / M_{t}\right\}$ is subnormal.

Proof. Obviously $S_{t} / M_{t}$ is subnormal when $S_{t}$ is subnormal. Assume that each $S_{t} / M_{t}$ is subnormal. By the Halmos-Bram criterion [2] to show that $S_{t}$ is subnormal we need only show that $\sum_{i, j=0}^{n}$ $\left(\mathrm{S}_{t}{ }^{i} f_{j}, \mathrm{~S}_{t}{ }^{j} f_{i}\right) \geqq 0$ for each finite collection $f_{0}, \cdots, f_{n}$. Since $\left\{\mathrm{S}_{t}\right\}$ is continuous we may assume that $t$ is rational. We further simplify our argument by assuming the $f_{j}$ to be continuous of compact support. There exist step functions $v_{j}(r), j=0, \cdots, n$ constant on the intervals $(m r,(m+1) r), m=0,1, \cdots$ and $r$ rational which uniformly approximate $f_{j} \mid \phi, j=0, \cdots, n$. Let $u_{j}{ }^{(r)}=\phi v_{j}{ }^{(r)}$ and note that the functions $u_{j}^{(r)}$ uniformly approximate $f_{j}, j=0, \cdots, n$ on the intervals $(m r,(m+1) r), m=0,1, \cdots$. Thus we need only show that $\sum_{i, j=0}^{n}\left(S_{t}{ }^{i} u_{j}{ }^{(r)}, S_{t}{ }^{j} u_{i}{ }^{(r)}\right) \geqq 0$ for each such collection $\left\{u_{j}{ }^{(r)}\right\}$. 
To this end we choose a positive integer $k$ such that $k(t / r)$ is integral and define $p(i)=i k(t / r)$. Also define $\tilde{u}_{p(i)}=u_{i}^{(r)}$ and $\tilde{u}_{m}=0$ if $m \neq p(i), i=0, \cdots, n$. Note that $\tilde{u}_{m} \in M_{r / k}$ for each $m$ and thus since $S_{r \mid k} / M_{r / k}$ is subnormal, then $0 \leqq \sum_{m, s \geqq 0}\left(S_{r \mid k}^{m} \tilde{u}_{s}, S_{r \mid k}^{s} \tilde{u}_{m}\right)$. But now we are through because

$$
\begin{aligned}
\sum_{m, s \geq 0}\left(S_{r / k}^{m} \tilde{u}_{s}, S_{r / k}^{s} \tilde{u}_{m}\right) & =\sum_{i, j=0}^{n}\left(S_{r / k}^{p(i)} u_{j}{ }^{(r)}, S_{r / k}^{p(j)} u_{i}{ }^{(r)}\right) \\
& =\sum_{i, j=0}^{n}\left(S_{t}{ }^{i} u_{j}{ }^{(r)}, S_{t}{ }^{j} u_{i}{ }^{(r)}\right) .
\end{aligned}
$$

THEOREM 3.2. $\left\{S_{t}\right\}$ is hyponormal if and only if $\left\{S_{t} / M_{t}\right\}$ is hyponormal.

Proof. The implication in one direction is obvious. Therefore we assume that each $S_{t} / M_{t}$ is hyponormal. To show that $S_{t}$ is hyponormal we need only show that $\sum_{i, j=0}^{1}\left(S_{t}^{i} f_{j}, S_{t}^{j} f_{i}\right) \geqq 0$ for all pairs $f_{0}, f_{1}$ (a moderately well-known but appàrently unpublished fact about hyponormal operators). We imitate the proof of Theorem 3.3 down to the point where we consider $\sum_{m, s \geqq 0}\left(S_{r / k}^{m} \tilde{u}_{s}, S_{r / k}^{s} \tilde{u}_{m}\right)$. We need to show this sum to be nonnegative. As previously observed it equals $\sum_{i, j=0}^{1}$ $\left(S_{r / k}^{p(i)} u_{j}^{(r)}, S_{r / k}^{p(j)} u_{i}^{(r)}\right)$ which is nonnegative because $S_{r / k}^{p(i)}$ is a power of a hyponormal weighted shift and hyponormal shifts enjoy the property that their powers are also hyponormal.

The second characterization of hyponormal semigroups $\left\{S_{t}\right\}$ is given by a geometric restriction on the symbol $\phi$. We show that $\left\{S_{t}\right\}$ is hyponormal only in case $\log \phi$ is convex. This gives us an extremely simple way in which to construct hyponormal $\left\{S_{t}\right\}$ : choose any continuous convex $\psi$ on $\mathrm{R}^{+}$; set $\phi=\exp \psi$ and construct the related semigroup $\left\{S_{t}\right\}$. To insure that $\left\{S_{t}\right\}$ is continuous we further require that $\psi(x+t)-\psi(x) \leqq w t$ for all $x$ and $t$ in $\mathbf{R}^{+}$and some $w$ (Lemma 2.1).

LEMMA 3.3. $\left\{\mathrm{S}_{t}\right\}$ is hyponormal if and only if $\log \phi$ is convex.

ProOF.

$$
\begin{aligned}
\left\|S_{t} f\right\|^{2}-\left\|S_{t}{ }^{*} f\right\|^{2}= & \int_{t}^{\infty} \frac{\phi(x)^{2}}{\phi(x-t)^{2}}|f(x-t)|^{2} d x \\
& -\int_{0}^{\infty} \frac{\phi(x+t)^{2}}{\phi(x)^{2}}|f(x+t)|^{2} d x
\end{aligned}
$$




$$
\begin{aligned}
= & \int_{0}^{t} \frac{\phi(x+t)^{2}}{\phi(x)^{2}}|f(x)|^{2} d x \\
& +\int_{t}^{\infty}\left(\frac{\phi(x+t)^{2}}{\phi(x)^{2}}-\frac{\phi(x)^{2}}{\phi(x-t)^{2}}\right)|f(x)|^{2} d x .
\end{aligned}
$$

Thus $S_{t}$ is hyponormal if and only if $\phi(x)^{2} \leqq \phi(x+t) \phi(x-t)$ for all $x$. This last inequality holds for all $x$ and $t$ if and only if $\log \phi(x) \leqq$ $(1 / 2)[\log \phi(x+t)+\log \phi(x-t)]$, or equivalently $\log \phi$ is convex.

THEOREM 3.1. and Lemma 3.3 enable us to construct an example of a hyponormal weighted translation semigroup which is not subnormal.

Example 3.4. $\left\{S_{t}\right\}$ is hyponormal but not subnormal. Define

Compute

$$
\phi(x)= \begin{cases}e^{-3 x+5}, & 0 \leqq x \leqq 1 \\ e^{-2 x+4}, & 1 \leqq x \leqq 2 \\ 1 & , \quad 2 \leqq x\end{cases}
$$

$$
\begin{aligned}
& \lambda_{0}^{2}=\int_{0}^{1} \phi^{2}=\frac{1}{6}\left(e^{10}-e^{4}\right) \\
& \lambda_{1}{ }^{2}=\int_{1}^{2} \phi^{2}=\frac{1}{4}\left(e^{4}-1\right) \\
& \lambda_{n}^{2}=\int_{n}^{n+1} \phi^{2}=1 \text { for } n \geqq 2 .
\end{aligned}
$$

This computation shows that the weights $\left\{\alpha_{i}\right\}$ associated with $S_{1} / M_{1}$ are such that $\alpha_{1}<\alpha_{2}<\alpha_{3}=\alpha_{4}=\cdots$. Thus by a theorem of Stampfli [8], $S_{1} / M_{1}$ is not subnormal and thus by Theorem 3.1 $\left\{S_{t}\right\}$ is not subnormal. On the other hand it is trivial to show that $\log \phi$ is convex and thus by Lemma $3.3\left\{\mathrm{~S}_{t}\right\}$ is hyponormal.

A brief reconsideration of the proof of Lemma 3.3 shows that none of the weighted translation semigroups can be normal. This follows from the fact that if $S_{t}$ is hyponormal and $\left\|S_{t} f\right\|=\left\|S_{t}{ }^{*} f\right\|$, then $\int_{0}^{t}\left(\phi(x+t)^{2} / \phi(x)^{2}\right)|f(x)|^{2} d x=0$. Therefore since $\phi \neq 0, f(x)=0$ a.e. on $[0, t]$; that is $f=0$ is the only function satisfying $\left\|S_{t} f\right\|=$ $\left\|S_{t}{ }^{*} f\right\|$ for all $t$.

On the other hand there do exist quasinormal semigroups. They are characterized by the symbols $\phi(x)=a e^{b x}, a, b$ constant, $a \neq 0$. We argue as follows: if $\left\{S_{t}\right\}$ is quasinormal, then $\left\|S_{t}{ }^{2} f\right\|=\left\|S_{t}{ }^{*} S_{t} f\right\|$ for all 
$f$ and $S_{t}$ is hyponormal. Thus from the proof of Lemma 3.3, $\int_{t}^{\infty}\left|\left(\phi(x+t)^{2} / \phi(x)^{2}\right)-\left(\phi(x)^{2} / \phi(x-t)^{2}\right)\right|\left|S_{t} f(x)\right|^{2} d x=0$ for all $f$. Equivalently $\phi(x+2 t) / \phi(x+t)=\phi(x+t) / \phi(x)$ for all $x$ and $t$, which implies that $\log \phi$ is linear. Therefore if $\left\{S_{t}\right\}$ is quasinormal, $\phi(x)=$ $a e^{b x}, a \neq 0$. The converse follows from a straightforward application of the definitions.

In the other direction we might hope for a more general class of semigroups by assuming only that $\left\{S_{t}\right\}$ is paranormal. We note that every hyponormal operator is paranormal, but the converse is not true in general. However we can show that every paranormal semigroup $\left\{S_{t}\right\}$ with symbol $\phi$ is hyponormal. To do this we invoke a theorem of Andô [1]: $A$ is paranormal if and only if $A^{* 2} A^{2}-2 \lambda A^{*} A$ $+\lambda^{2} \gg 0$ for all $\lambda>0$. Applied to our semigroup this results in the relation $\int_{0}^{\infty}\left[\left(\phi(x+2 t)^{2} / \phi(x)\right)-2 \lambda\left(\phi(x+t)^{2} / \phi(x)^{2}\right)+\lambda^{2}\right]$ $|f(x)|^{2} d x \geqq 0$ for all $f$ in $L^{2}$ and $\lambda>0$. Consequently for all $\lambda>0$ and $x$ and $t$ in $\mathbf{R}^{+},\left(\phi(x+2 t)^{2} / \phi(x)^{2}\right)-2 \lambda\left(\phi(x+t)^{2} / \phi(x)^{2}\right)+\lambda^{2}$ $\geqq 0$. This occurs only if $\left(\phi(x+t)^{4} / \phi(x)^{4}\right) \leqq\left(\phi(x+2 t)^{2} / \phi(x)^{2}\right)$; that is, $\log \phi$ is convex and $\left\{S_{t}\right\}$ is hyponormal.

The preceding remarks give us strong evidence that the weighted translation semigroups have peculiar properties. If $\left\{S_{t}\right\}$ is an arbitrary hyponormal semigroup, then its infinitesimal generator $G$ is hyponormal in the sense that $\|G f\| \geqq\left\|G^{*} f\right\|$ for all $f$ in $D(G) \cap D\left(G^{*}\right)$. (Indeed, $\quad\|G f\|=\lim _{t \rightarrow 0}\left\|S_{t} f-f\right\| / t \geqq \lim _{t \rightarrow 0}\left\|S_{t}{ }^{*} f-f\right\| / t=\left\|G^{*} f\right\|$.) It is not reasonable to assume that the converse is true because there are many examples of hyponormal operators for which certain algebraic combinations are not hyponormal. However, we shall show in Theorem 3.6 that a weighted translation semigroup with a $C^{1}$ symbol $\phi$ is hyponormal in case its generator is hyponormal.

It is known [5] that if $\left\{S_{t}\right\}$ is subnormal in the sense that each $S_{t}$ is subnormal, then $\left\{S_{t}\right\}$ is subnormal in the seemingly stronger sense that there exists a normal semigroup $\left\{N_{t}\right\}$ defined on a Hilbert space $Y$, containing $X$, such that $N_{t} / X=S_{t}$. It follows easily now that $\{S t\}$ is subnormal if and only if its generator $G$ is subnormal. Therefore we learn nothing new along these lines by assuming that $\left\{S_{t}\right\}$ is a weighted translation semigroup.

By Lemma 3.3 we know that $\log \phi$ is convex if $\left\{S_{t}\right\}$ is hyponormal. Thus $\log \phi$ is continuous and differentiable almost everywhere [9; p. 134]. Consequently $\phi$ is differentiable almost everywhere. Moreover, since $\left\{S_{t}\right\}$ is continuous, $\phi(x+t) / \phi(x) \leqq e^{w t}$ for all $x$ and $t$ and some $w$. This implies that $\phi^{\prime}(x) / \phi(x) \leqq w$ whenever $\phi^{\prime}(x)$ exists. The hypotheses in the next lemma and theorem are a slight strengthening of these conditions. 
LeMma 3.5. Assume that $\phi$ is a $C^{1}$ function on $(0, \infty)$ and $\phi^{\prime} / \phi \in$ $L^{\infty}$. Then the infinitesimal generators $G$ of $\left\{S_{t}\right\}$ and $G^{*}$ of $\left\{S_{t}^{*}\right\}$ are given by

(i) $D(G)=\left\{f: f \in L^{2}, f\right.$ absolutely continuous, $f^{\prime} \in$ $\left.L^{2}, f(0)=0\right\}$ and $G f=-f^{\prime}+\left(\phi^{\prime} \mid \phi\right) f$

(ii) $D\left(G^{*}\right)=\left\{f: f \in L^{2}, f\right.$ absolutely continuous, $f^{\prime}$ $\left.\in L^{2}\right\}$ and $G^{*} f=f^{\prime}+\left(\phi^{\prime} / \phi\right) f$.

Proof. By definition $D(G)$ is the set of $f$ in $L^{2}$ for which $\lim _{t \rightarrow 0} S_{t} f-f / t$ exists in the $L^{2}$ norm and in this case $G f$ is the limit. For such $f$, the pointwise limit exists for a subsequence of $t \rightarrow 0$ and thus we have for $x>0$

$$
\begin{aligned}
G f(x) & =\lim _{t \rightarrow 0} \frac{S_{t} f(x)-f(x)}{t} \\
& =\lim _{t \rightarrow 0} \frac{1}{t}\left(\frac{\phi(x)}{\phi(x-t)} f(x-t)-f(x)\right) .
\end{aligned}
$$

By elementary techniques this last limit equals $-f^{\prime}(x)+\phi^{\prime}(x) / \phi(x)$ $f(x)$. For $\lambda$ sufficiently large we have

$$
\begin{aligned}
D(G) & =\operatorname{range}(\lambda-G)^{-1} \\
& =\left\{\int_{0}^{\infty} e^{-\lambda t} S_{t} g d t: g \in L^{2}\right\} .
\end{aligned}
$$

Thus $D(G)$ consists precisely of functions of the form

$$
f(x)=\frac{\phi(x)}{e^{\lambda x}} \int_{0}^{x} \frac{e^{\lambda t}}{\phi(t)} g(t) d t \quad \text { a.e.; } g \in L^{2} .
$$

Hence, since $\phi$ is in $C^{\prime}\left(\mathbf{R}_{+}\right), f$ is absolutely continuous and $f(0)=0$. Moreover, since $f^{\prime}=-G f-\left(\phi^{\prime} / \phi\right) f$ and $\left(\phi^{\prime} / \phi\right) L^{2} \subset L^{2}, f^{\prime}$ is in $L^{2}$. We have shown that $D(G) \subset\left\{f: f \in L^{2}, f\right.$ absolutely continuous, $\left.f^{\prime} \in L^{2}, f(0)=0\right\}$ and for $f$ in $D(G), G f=-f^{\prime}+\left(\phi^{\prime} \mid \phi\right) f$. Let $K=$ $-D+M_{\phi^{\prime} \mid \phi}$ where $D$ is the differentiation operator on the described set. $K$ is an infinitesimal generator since $K$ is the perturbation of the generator $-D$ by the bounded $\left(\phi^{\prime} / \phi \in L^{\infty}\right)$ operator $M_{\phi^{\prime} / \phi}([6 ; \mathrm{p}$. 495]). Since infinitesimal generators are maximal, $G=K$ as desired. Since $M_{\phi^{\prime} \mid \phi}$ is bounded and self adjoint, $G^{*}=K^{*}=-D^{*}+M$, and so $D\left(G^{*}\right)=D\left(D^{*}\right)=\left\{f: f \in L^{2}, f\right.$ absolutely continuous, $f^{\prime} \in$ $\left.L^{2}\right\}$. 
THEOREM 3.6. Assume that $\phi$ is a $C^{1}$ function and $\phi^{\prime} / \phi \in L^{\infty}$. Then $\left\{S_{t}\right\}$ is hyponormal if and only if its infinitesimal generator $G$ is hyponormal.

Proof. In the remarks preceding Lemma 3.5 we proved the necessity of the condition. Assume then that $G$ is hyponormal. By Lemma 3.5 for all $f$ in $D(G)$ we have

$$
\begin{aligned}
0 & \leqq\|G f\|^{2}-\left\|G^{*} f\right\|^{2} \\
& =\int\left|-f^{\prime}+\left(\phi^{\prime} \mid \phi\right) f\right|^{2}-\int\left|f^{\prime}+\left(\phi^{\prime} \mid \phi\right) f\right|^{2} \\
& =-2 \int\left(\phi^{\prime} / \phi\right) \operatorname{Re} f^{\prime} \bar{f} .
\end{aligned}
$$

That is, $\int\left(\phi^{\prime} / \phi\right) \operatorname{Re} f^{\prime} \bar{f} \leqq 0$ for all $f$ in $D(G)$. If we can show that this condition implies that $\log \phi$ is convex, then by Lemma $3.3\left\{\mathrm{~S}_{t}\right\}$ is hyponormal. Let $0 \leqq a<b$. Define $f$ by

Then $f \in D(G)$ and

$$
f(x)= \begin{cases}0 & \text { if } x \leqq a \\ x-a & \text { if } a \leqq x \leqq(1 / 2)(a+b) \\ b-x & \text { if }(1 / 2)(a+b) \leqq x \leqq b \\ 0 & \text { if } b \leqq x\end{cases}
$$

$$
\int \frac{\varphi^{\prime}}{\varphi} \operatorname{Re} f^{\prime} \bar{f}=(b-a) \log \phi\left(\frac{1}{2}(a+b)\right)-\int_{a}^{b} \log \phi .
$$

Therefore on each interval $[a, b]$

$$
(b-a) \log \phi\left(\frac{1}{2}(a+b)\right) \leqq \int_{a}^{b} \log \phi .
$$

Equivalently $\log \phi$ is convex; the proof is complete.

Answers to the following questions would be of value in the investigation of the structure of weighted translation semigroups.

1. What conditions on $\phi$ insure the subnormality of $\left\{S_{t}\right\}$ ?

2. If $\left\{S_{t}\right\}$ and $\left\{T_{t}\right\}$ are weighted translation semigroups with symbols $\phi$ and $\rho$ respectively, and if $\left\{S_{t}\right\}$ is similar to $\left\{T_{t}\right\}$ (i.e., there is an invertible operator $A$ such that $A S_{t}=T_{t} A$ for all $t$ ), does it necessarily follow that $|\rho / \phi|$ is bounded above and below? (This boundedness condition does imply similarity.).

3. Can the hypotheses regarding $\phi$ be weakened in Theorem 3.6? 


\section{REFERENCES}

1. T. Andô, Operators with a norm condition, Acta Scientiarum Mathematicarum (Szeged), 33 (1972), 169-178.

2. J. Bram, Subnormal operators, Duke Math. J. 22 (1955), 75-94.

3. N. Dunford and J. T. Schwartz, Linear Operators, Part I, Interscience Publishers, Inc., New York, N.Y.: 1967.

4. P. R. Halmos, A Hilbert Space Problem Book, Van Nostrand, Princeton, N.J.: 1967.

5. T. Ito, On the commutative family of subnormal operators, J. Fac. Sci., Hokkaido Univ., 14 (1958), 1-15.

6. T. Kato, Perturbation Theory for Linear Operators, Springer-Verlag, New York, N.Y.: 1966.

7. R. L. Kelley, Weighted shifts on Hilbert space, Dissertation, Univ. of Mich.: 1966.

8. J. G. Stampfli, Which weighted shifts are subnormal? Pacific J. Math. 17 (1966), 367-379.

9. F. A. Valentine, Convex Sets, McGraw-Hill, New York, N.Y.: 1964.

University of North Carolina at Charlotte, Charlotte, North Carolina 